\title{
A STOCHASTIC MODEL FOR THE FINANCIAL MARKET WITH DISCONTINUOUS PRICES ${ }^{1}$
}

\author{
LEDA D. MINKOVA \\ Technical University of Sofia \\ Institute of Applied Mathematics and Informatics \\ P.O. Box 384, 1000 Sofia, Bulgaria
}

(Received May, 1994; Revised January, 1996)

\begin{abstract}
This paper models some situations occurring in the financial market. The asset prices evolve according to a stochastic integral equation driven by a Gaussian martingale. A portfolio process is constrained in such a way that the wealth process covers some obligation. A solution to a linear stochastic integral equation is obtained in a class of cadlag stochastic processes.
\end{abstract}

Key words: Contingent Claim Valuation, Representation of Martingales, Stochastic Integral Equation, Option Pricing, Portfolio Processes.

AMS (MOS) subject classifications: $60 \mathrm{H} 20,60 \mathrm{H} 30$.

\section{Introduction}

In the present paper we model investments of an economic agent whose decisions cannot affect market prices (a "small investor").

Karatzas and Shreve in [7] considered a market model in which prices evolve according to a stochastic differential equation, driven by Brownian motion. Aase [1] and M. Picqué and M. Pontier [9] studied a more general model in which the evolution of asset prices is a combination of a continuous process based on Brownian motion (a semimartingale) and a Poisson point process.

The security price model that we use is a linear stochastic equation driven by a Gaussian martingale. This is a natural generalization, because the market is not continuous and the Brownian motion cannot model jump processes. Moreover, the instants of jumps of a Gaussian martingale are nonrandom.

The techniques we use include the martingale representation theorem and the Girsanov's type theorem. We also find a solution to a linear stochastic integral equation.

\section{The Model}

We consider a model of a security market where an economic agent is allowed to trade continuously up to some fixed planning horizon $0 \leq T<\infty$. We shall denote by $X_{t}$ the wealth of this

\footnotetext{
${ }^{1}$ This work was supported by the National Science Foundation of Bulgaria, Grant No.
} MM-440/94. 
agent at time $t$. Let the process $\mathbf{M}=\left(M_{t}, F_{t}, 0 \leq t \leq T\right)$ be a Gaussian martingale on a fixed probability space $(\Omega, F, \mathbf{P})$ and the filtration $\mathbf{F}=\left\{F_{t}, 0 \leq t \leq T\right\}$ be the augmentation under $\mathbf{P}$ of a natural filtration $F_{t}^{M}=\sigma\left(M_{s}, 0 \leq s \leq t\right), 0 \leq t<\infty$. $F_{0}$ contains the null sets of $\mathbf{P}$ and $\mathbf{F}$ is right continuous. $\langle M\rangle_{t}=E M_{t}^{2}, t \in \mathbb{R}_{+}=[0, \infty)$ is the square characteristic of $\mathbf{M}$.

Let us suppose that the agent invests in two assets (or "securities"). One of the assets, called bond, has a finite variation on $[0, T]$, and its price model is

$$
P_{0}(t)=\int_{(0, t]} P_{0}(s-) r(s-) d\langle M\rangle_{s}, \quad P_{0}(0)=p_{0}, 0 \leq t \leq T .
$$

The other one, called stock, is "risky". Its price is modeled by the linear stochastic equation

$$
P(t)=\int_{(0, t]} P(s-) A(s-) d\langle M\rangle_{s}+\int_{(0, t]} P(s-) \sigma(s-) d M_{s}, \quad P(0)=p .
$$

Here the interest rate process $r(t)>0,0 \leq t<\infty$ of the bond, the appreciation rate process $A(t)$ of the stock, and volatility process $\sigma(t)>0,0 \leq t<\infty$ will all be nonrandom, $F$-predictable processes such that

$$
\begin{gathered}
\int_{(0, \infty)} r^{2}(s-) d\langle M\rangle_{s}<\infty, \int_{(0, \infty)} A^{2}(s-) d\langle M\rangle_{s}<\infty, \\
\int_{(0, \infty)} \sigma^{2}(s-) d\langle M\rangle_{s}<\infty, \text { P-a.s. }
\end{gathered}
$$

In addition, $A(t-) \Delta\langle M\rangle_{t}+\sigma(t-) \Delta M_{t}>-1, t \in(0, T]$, to ensure a limited liability of the stock. is

Let $m(t)$ denote the number of stocks held at time $t$. Then the amount invested in the stocks

$$
\Pi(t)=m(t) P(t)
$$

The process $\left(\Pi(t), F_{t}\right), 0 \leq t \leq T$ describes the investment policy and will be called a portfolio process. It is assumed to be measurable, $F_{t}$-predictable and

$$
\int_{(0, T]} \Pi^{2}(s-) d\langle M\rangle_{s}<\infty, \text { P-a.s. }
$$

for every finite number $T>0$. Note that $\Pi(t)$ can be negative, which amounts to selling the stock short.

On the other hand, $C(t), 0 \leq t \leq T$ is a non-negative consumption process, assumed to be nondecreasing and $F_{t}$-predictable, such that

$$
\int_{(0, T]} C(s-) d\langle M\rangle_{s}<\infty, \text { P-a.s. }
$$

for every finite number $T>0$.

The quantity

$$
\Pi_{0}(t)=X_{t}-\Pi(t)
$$

is invested in the bond at any particular time and may also become negative. This is to be interpreted as borrowing at the interest rate $r(t)$. 
We assume now that the investor starts with some initial wealth $x \geq 0$, and the wealth at time $t$ satisfies the linear stochastic equation

$$
\begin{gathered}
X_{t}=\int_{(0, t]} \Pi(s-) \sigma(s-) d M_{s}+\int_{(0, t]} \Pi(s-)[A(s-)-r(s-)] d\langle M\rangle_{s} \\
+\int_{(0, t]}\left[X_{s-} r(s-)-C(s-)\right] d\langle M\rangle_{s}, \quad 0<t \leq T ; \\
X(0)=x .
\end{gathered}
$$

Conditions (1), (2), and (3) ensure that the stochastic equation (4) has a unique solution in the class of cadlag adapted processes (see Section 5 and Theorem 3 ).

\section{Characterization of the Portfolio Process}

If $A(t)=r(t)$ for every $t \in[0, \infty)$, the drift

$$
\int_{(0, t]} \Pi(s-)[A(s-)-r(s-)] d\langle M\rangle_{s}
$$

vanishes from the right-hand side of (4). When $A(t) \not \equiv r(t)$ we introduce a new probability measure $\overline{\mathbf{P}}$ which removes this drift.

Let us denote by $\phi_{t}$ the solution of the equation

where

$$
\phi_{t}=1-\int_{(0, t]} \phi_{s-} \theta(s-) d M_{s}, \quad 0 \leq t \leq T
$$

$$
\theta(t)=\frac{A(t)-r(t)}{\sigma(t)}
$$

From our assumptions on $A, r$, and $\sigma$, it follows that $\theta(t)$ is bounded, measurable and adapted to $\left\{F_{t}-\right\}$. Then the exponential supermartingale

$$
\phi_{t}=\exp \left[-\int_{(0, t]} \theta(s-) d M_{s}^{c}-\frac{1}{2} \int_{(0, t]} \theta^{2}(s-) d\left\langle M^{c}\right\rangle_{s}\right] \cdot \prod_{s \leq t}\left[1-\theta(s-) \Delta M_{s}\right]
$$

is actually a martingale, where

$$
\Delta M_{t}=M_{t}-M_{t}-\not \equiv \frac{\sigma(t-)}{A(t-)-r(t-)} \quad \text { for } 0<t \leq T .
$$

Here $M_{t}^{c}$ and $\left\langle M^{c}\right\rangle_{t}$ are the continuous parts of the processes $M_{t}$ and $\langle M\rangle_{t}$, respectively, for $t \in$ $\mathbb{R}_{+}$.

We define the new probability measure $\overline{\mathbf{P}}$ :

$$
\overline{\mathbf{P}}(A)=\mathbf{E}\left(\phi_{T} \mathbf{I}_{A}\right), \quad A \in F_{T} \text { on }(\Omega, F) .
$$

The probability measures $\mathbf{P}$ and $\overline{\mathbf{P}}$ are mutually absolutely continuous on $F_{T}$.

The process

$$
\bar{M}_{t}=M_{t}+\int_{(0, t]} \theta(s-) d\langle M\rangle_{s}, \quad 0 \leq t \leq T
$$

is a $\overline{\mathbf{P}}$-Gaussian martingale [8], and

$$
\left(\langle\bar{M}\rangle_{t}, \overline{\mathbf{P}}\right) \equiv\left(\langle M\rangle_{t}, \mathbf{P}\right), \quad 0 \leq t \leq T .
$$


With respect to a new probability measure, equation (4) can be rewritten as

$$
\begin{gathered}
X_{t}=\int_{(0, t]} \Pi(s-) \sigma(s-) d \bar{M}_{s}+\int_{(0, t]}\left[X_{s-} r(s-)-C(s-)\right] d\langle\bar{M}\rangle_{s}, 0<t \leq T, \\
X(0)=x
\end{gathered}
$$

and the solution (see Section 5 ) for $0 \leq t \leq T$, leads to

where

$$
\frac{X_{t}}{\Phi(t)}+\int_{(0, t]} \frac{C(s-)}{\Phi(s-)\left[1+r(s-) \Delta\langle M\rangle_{s}\right]} d\langle\bar{M}\rangle_{s}=x+\int_{(0, t]} \frac{\Pi(s-) \sigma(s-)}{\Phi(s-)\left[1+r(s-) \Delta\langle M\rangle_{s}\right]} d \bar{M}_{s}
$$

$$
\Phi(t)=\exp \left[\int_{(0, t]} r(s-) d\left\langle M^{c}\right\rangle_{s}\right] \cdot \prod_{s \leq t}\left[1+r(s-) \Delta\langle M\rangle_{s}\right]
$$

is a unique strong solution of the homogeneous equation corresponding to (7):

$$
\Phi(t)=1+\int_{(0, t]} \Phi(s-) r(s-) d\langle M\rangle_{s} .
$$

If we suppose that $1+r(s-) \Delta\langle M\rangle_{s} \leq 0$ for some $s \in \mathbb{S}$, then $\Delta\langle M\rangle_{s} \leq-\frac{1}{r(s-)}$. But this is impossible if $r(s)$ is nonnegative. Consequently, $1+r(s-) \Delta\langle M\rangle_{s}>0$ for every $s \in \mathbb{R}_{+}$.

Let us notice also that

$$
\inf _{t \in \mathbb{R}_{+}}|\Phi(t)|>0
$$

The right-hand side of (8) is a $\overline{\mathbf{P}}$-local martingale. If $(\Pi, C)$ is an admissible pair (i.e., $X_{t} \geq$ $0,0 \leq t \leq T$ a.s.), the left-hand side is nonnegative, consequently it is a nonnegative supermartingale under $\overline{\mathbf{P}}$. From the supermartingale property we obtain that

$$
\overline{\mathbf{E}}\left[\frac{X_{T}}{\Phi(T)}+\int_{(0, T]} \frac{C(s-)}{\Phi(s-)\left[1+r(s-) \Delta\langle M\rangle_{s}\right]} d\langle\bar{M}\rangle_{s}\right] \leq x
$$

where $\overline{\mathbf{E}}$ denotes the expectation operator under measure $\overline{\mathbf{P}}$.

This condition is also sufficient for the admissibility in the sense of the following theorem.

Theorem 1: Suppose that $x \geq 0$ and $B_{T}$ is a nonnegative $F_{T}$-measurable random variable, such that

$$
\overline{\mathbf{E}}\left[\frac{B_{T}}{\Phi(T)}+\int_{(0, T]} \frac{C(s-)}{\Phi(s-)\left[1+r(s-) \Delta\langle M\rangle_{s}\right]} d\langle\bar{M}\rangle_{s}\right] \leq x .
$$

Then there exists a portfolio process $\Pi$ such that the pair $(\Pi, C)$ is admissible for the initial endowment $x$ and the terminal wealth $X_{T}$ is at least $B_{T}$.

Proof: It is obvious that we can assume equality to hold in (11).

Let us define the nonnegative process

$$
\begin{gathered}
\bar{\mu}_{t}=\overline{\mathbf{E}}\left[\frac{B_{T}}{\Phi(T)}+\int_{(0, T]} \frac{C(s-)}{\Phi(s-)\left[1+r(s-) \Delta\langle M\rangle_{s}\right]} d\langle\bar{M}\rangle_{s} \mid F_{t}\right] \\
\bar{\mu}_{0}=x
\end{gathered}
$$


which is a $\overline{\mathbf{P}}$-martingale and has "cadlag" paths.

Define the process $\mu_{t}, 0 \leq t \leq T$ by

$$
\mu_{t}=\bar{\mu}_{t}+\int_{(0, t]} \phi_{s-1}^{-1} d\langle\phi, \bar{\mu}\rangle_{s}
$$

where $\phi_{t}$ is the density (5). It is well known that the process $\mu_{t}$ is a P-martingale [5], $\mu_{0}=\bar{\mu}_{0}$, and $\langle\mu\rangle=\langle\bar{\mu}\rangle$.

Now by the martingale representation theorem [8], if $(\mathbf{M}, \mu)$ is a Gaussian process, there exists an $F_{t}$-predictable measurable process $h(s)$, such that

for every finite $T>0$ and

$$
\int_{(0, T]} h^{2}(s-) d\langle M\rangle_{s}<\infty, \quad \text { P-a.s. }
$$

$$
\mu_{t}=\mu_{0}+\int_{(0, t]} h(s-) d M_{s}, \quad 0 \leq t \leq T .
$$

The process (13) can be represented as

$$
\mu_{t}=\bar{\mu}_{t}-\int_{(0, t]} \theta(s-) h(s-) d\langle M\rangle_{s}, \quad 0 \leq t \leq T .
$$

From equalities (6), (14), and (15) it follows that

$$
\begin{gathered}
\bar{\mu}_{t}=\mu_{t}+\int_{(0, t]} \theta(s-) h(s-) d\langle M\rangle_{s} \\
=\mu_{0}+\int_{(0, t]} h(s-)\left[d \bar{M}_{s}-\theta(s-) d\langle M\rangle_{s}\right]+\int_{(0, t]} \theta(s-) h(s-) d\langle M\rangle_{s} \\
=\mu_{0}+\int_{(0, t]} h(s-) d \bar{M}_{s} .
\end{gathered}
$$

Now,

$$
\Pi(t-)=\frac{h(t-) \Phi(t-)\left[1+r(t-) \Delta\langle M\rangle_{t}\right]}{\sigma(t-)}, 0<t \leq T
$$

is a well-defined portfolio process.

From (12), (16), and (17), we get

$$
\begin{gathered}
\bar{\mu}_{t}=\overline{\mathbf{E}}\left[\frac{B_{T}}{\Phi(T)}+\int_{(0, T]} \frac{C(s-)}{\Phi(s-)\left[1+r(s-) \Delta\langle M\rangle_{s}\right]} d\langle\bar{M}\rangle_{s} \mid F_{t}\right] \\
=x+\int_{(0, t]} h(s-) d \bar{M}_{s} .
\end{gathered}
$$

By using (18) and (8), we obtain

$$
\bar{\mu}_{t}=\frac{X_{t}^{\Pi, C, x}}{\Phi(t)}+\int_{(0, t]} \frac{C(s-)}{\Phi(s-)\left[1+r(s-) \Delta\langle M\rangle_{s}\right]} d\langle\bar{M}\rangle_{s}
$$


where $X_{t}^{\Pi, C, x}$ is a solution of equation (7) for the pair (I, $C$ ) and the initial capital $x \geq 0$.

Now, from (18) and (19), it follows that

$$
\frac{X_{t}^{\Pi, C, x}}{\Phi(t)}=\overline{\mathbf{E}}\left[\frac{B_{T}}{\Phi(T)}+\int_{(t, T]} \frac{C(s-)}{\Phi(s-)\left[1+r(s-) \Delta\langle M\rangle_{s}\right.} d\langle\bar{M}\rangle_{s} \mid F_{t}\right] .
$$

Consequently, $X_{t}^{\Pi, C, x}$ is nonnegative and $(\Pi, C)$ is an admissible strategy.

\section{Valuation of Contingent Claim}

Definition: A contingent claim is a nonnegative $F_{T}$-measurable random variable $B$ that satisfies

$$
0<\overline{\mathbf{E}}\left[\frac{B}{\Phi(T)}\right] \leq x
$$

The hedging price of this contingent claim is defined by

$$
U \stackrel{\text { def }}{=} \inf \left\{x>0, \exists(\Pi, C) \text { - admissible, such that } X_{T}^{\Pi, C, x} \geq B \text { P-a.s. }\right\} \text {. }
$$

Theorem 2: The value of the contingent claim is attained and

$$
U=\overline{\mathbf{E}}\left[\frac{B}{\Phi(T)}\right]
$$

Proof: Let us suppose that $X_{T}^{\Pi, C, x} \geq B$ a.s. for some value of $x>0$ and a suitable pair $(\Pi, C)$. Then from $(10)$ it follows that

Consequently, $z=\overline{\mathbf{E}}\left[\frac{B}{\Phi(T)}\right] \leq U$.

$$
\overline{\mathbf{E}}\left[\frac{B}{\Phi(T)}\right] \leq \overline{\mathbf{E}}\left[\frac{X_{T}^{\Pi, C, x}}{\Phi(T)}\right] \leq x
$$

Let us define the nonnegative random process

$$
X_{0}(t)=\Phi(t) \cdot \overline{\mathbf{E}}\left[\frac{B}{\Phi(T)} \mid F_{t}\right], \quad 0 \leq t \leq T
$$

where $\bar{m}_{t}=\overline{\mathbf{E}}\left[\frac{B}{\Phi(T)} \mid F_{t}\right]$ is a $\overline{\mathbf{P}}$-Gaussian martingale, such that $\bar{m}_{0}=\overline{\mathbf{E}}\left[\frac{B}{\Phi(T)}\right]$.

Analogously to the proof of the Theorem 1, we can apply the generalized Girsanov's theorem and the martingale representation theorem.

By comparing the processes

and $\frac{X_{t}^{\Pi, 0, x}}{\Phi(t)}$ we obtain that

$$
\begin{gathered}
\frac{X_{0}(t)}{\Phi(t)}=z+\int_{(0, t]} h(s-) d \bar{M}_{s} \\
X_{0}(t) \equiv X_{t}^{\Pi, 0, z}, \quad 0 \leq t \leq T .
\end{gathered}
$$

Consequently, $z \geq U$.

Remark 1: Let us note that (21) yields

$$
X_{0}(T) \equiv X_{T}^{\Pi, 0, z}=B, \text { a.s. }
$$

i.e., the contingent claim is attained with the initial capital $U$, portfolio $\Pi$, and zero consumption. This fact could be used as a starting point for solving appropriate optimal problems. 
Remark 2: If $\langle M\rangle_{t} \equiv t$, we have $(\mathbf{M}, \mathbf{P})$ and $(\overline{\mathbf{M}}, \overline{\mathbf{P}})$ (standard) Wiener processes, and $\mathbb{S}$ empty. Then Theorem 1 and Theorem 2 reduce to the results of Karatzas and Shreve [7] and Cvitanić and Karatzas [2].

Corollary: Let $C(t) \equiv 0$ and let the agent invest in one stock asset. Then, the following representations hold:

$$
\begin{gathered}
X_{t}=\Phi(t) \cdot \overline{\mathbf{E}}\left[\frac{P(T)}{\Phi(T)} \mid F_{t}\right], \quad 0 \leq t \leq T \\
X_{t}=p \cdot \exp \left[\int_{(0, t]} \sigma(s-) d \bar{M}_{s}^{c}-\frac{1}{2} \int_{(0, t]} \sigma^{2}(s-) d\left\langle\bar{M}^{c}\right\rangle_{s}+\int_{(0, t]} r(s-) d\left\langle\bar{M}^{c}\right\rangle_{s}\right] \\
\cdot \prod_{s \leq t}\left[1+r(s-) \Delta\langle\bar{M}\rangle_{s}+\sigma(s-) \Delta \bar{M}_{s}\right], \quad 0 \leq t \leq T .
\end{gathered}
$$

Proof: Representation $(i)$ follows from $(20)$ when $C(t) \equiv 0$.

By Ito's rule it can be proved that $\frac{P(T)}{\Phi(T)}$ is a $\overline{\mathbf{P}}$-Gaussian martingale and is a unique solution of the following stochastic equation:

$$
\frac{P(T)}{\Phi(T)}=p+\int_{(0, T]} \frac{P(s-)}{\Phi(s-)} \cdot \frac{\sigma(s-)}{\left[1+r(s-) \Delta\langle M\rangle_{s}\right]} d \bar{M}_{s}, \quad T>0 .
$$

Consequently, from representation $(i)$ it follows that $\frac{X_{t}}{\Phi(t)}, 0 \leq t \leq T$ is a $\overline{\mathbf{P}}$-Gaussian martingale and it yields representation $(i i)$.

\section{A Linear Stochastic Integral Equation}

In this section we will obtain a solution of the stochastic equation

$$
X_{t}=X_{0}+\int_{(0, t]}\left[S(s) X_{s-}+\sigma(s)\right] d M_{s}+\int_{(0, t]}\left[A(s) X_{s-}+a(s)\right] d b(s),
$$

$0 \leq t<\infty$, which is a more general than we anticipate.

Let $\mathbf{M}=\left(M_{t}, F_{t}\right), M_{0}=0, F_{t}=\sigma\left(M_{s}, s \leq t\right), t \in \mathbb{R}_{+}=[0, \infty)$, be a cadlag Gaussian martingale, $b=b(t), t \in \mathbb{R}_{+}$-nonrandom, right-continuous function with finite variation on each finite interval. Suppose the function $b(t)$ be a real-valued deterministic function, absolutely continuous with respect to $\langle M\rangle_{t}=\mathbf{E} M_{t}^{2}$ and

$$
b(t)=\int_{(0, t]} \gamma_{s} d\langle M\rangle_{s}, \quad t \in \mathbb{R}_{+},
$$

where $\gamma=\left(\gamma_{t}, F_{t-}\right), F_{t-}=\sigma\left(M_{s}, 0<s<t\right)$ is a $F$-predictable function, $X_{0}=\left(X_{0}, F_{0}\right)$ be a Gaussian random variable, independent of M. $A(t), \sigma(t), a(t)$, and $S(t)$ are nonrandom $F$-predictable functions, such that

$$
\begin{gathered}
\int_{(0, \infty)} \sigma^{2}(s) d\langle M\rangle_{s}<\infty, \quad \int_{(0, \infty)} A^{2}(s) d b(s)<\infty \\
\int_{(0, \infty)} a^{2}(s) d b(s)<\infty \quad \text { and } \quad \int_{(0, \infty)} S^{2}(s) d b(s)<\infty, \text { P-a.s. }
\end{gathered}
$$

We will find a solution of equation (22) in the class of cadlag adapted processes (i.e., pro- 
cesses with right-continuous paths and finite left limits) and it provides a fairly explicit representation. According to [4], such a solution exists and it is unique in the sense of P-indistinguishability.

Recall [6] that the random process $\mathbf{M}$ and the deterministic nondecreasing function $\langle M\rangle$ have their jumps at the same nonrandom moments of time which form a countable set $\mathbb{S} \subset \mathbb{R}_{+} \backslash\{0\}$. Let us notice now that the function $b(t)$ and the process $X_{t}$ have their jumps at the same moments.

Let us suppose that the function $b=b(t), t \in \mathbb{R}_{+}$has no more than a countable subset of jumps $\left\{0 \leq s_{0}<s_{1}<\ldots<s_{k}<\ldots<\infty\right\} \subseteq \mathbb{S}$ with

It is obvious that

$$
\Delta b\left(s_{k}\right)=-\frac{1+S\left(s_{k}\right) \Delta M_{s_{k}}}{A\left(s_{k}\right)}, k \geq 1 .
$$

$$
\begin{aligned}
\Delta X_{s_{k}} & =\left[A\left(s_{k}\right) X_{s_{k}}+a\left(s_{k}\right)\right] \Delta b\left(s_{k}\right)+\left[S\left(s_{k}\right) X_{s_{k}}+\sigma\left(s_{k}\right)\right] \Delta M_{s_{k}} \\
& =-X_{s_{k}}-\frac{a\left(s_{k}\right)}{A\left(s_{k}\right)}\left[1+S\left(s_{k}\right) \Delta M_{s_{k}}\right]+\sigma\left(s_{k}\right) \Delta M\left(s_{k}\right) .
\end{aligned}
$$

Consequently,

$$
X_{s_{k}}=\sigma\left(s_{k}\right) \Delta M_{s_{k}}-\frac{a\left(s_{k}\right)}{A\left(s_{k}\right)}\left[1+S\left(s_{k}\right) \Delta M_{s_{k}}\right]
$$

We will find a solution of equation (22) on the interval $\left[s_{k}, s_{k+1}\right), k \geq 0$, with an initial condition $X_{s_{k}}$, independent of increments $M_{t}-M_{s_{k}}, s_{k} \leq t<s_{k+1}, k \geq 0$, according to (23) and the conditions imposed on $X_{0}$.

The homogeneous equation corresponding to (22) is

$$
\begin{gathered}
\Phi\left(t, s_{k}\right)=1+\int_{\left(s_{k}, t\right]} \Phi\left(s-, s_{k}\right) A(s) d b(s)+\int_{\left(s_{k}, t\right]} \Phi\left(s-,{ }_{k}\right) S(s) d M_{s}, \\
\Phi\left(s_{k}, s_{k}\right)=1, \quad k \geq 0
\end{gathered}
$$

and has a unique solution [3]:

$$
\begin{gathered}
\Phi\left(t, s_{k}\right)=\exp \left\{\int_{\left(s_{k}, t\right]} A(s) d b(s)+\int_{\left(s_{k}, t\right]} S(s) d M_{s}-\frac{1}{2} \int_{\left(s_{k}, t\right]} S^{2}(s) d\left\langle M^{c}\right\rangle_{s}\right\} \\
\cdot \prod_{s_{k}<s \leq t}\left\{1+A(s) \Delta b(s)+S(s) \Delta M_{s}\right\} \cdot \exp \left\{-A(s) \Delta b(s)-S(s) \Delta M_{s}\right\} ; \\
\Phi\left(t, s_{k}\right)=\exp \left\{\int_{\left(s_{k}, t\right]} A(s) d b^{c}(s)+\int_{\left(s_{k}, t\right]} S(s) d M_{s}^{c}-\frac{1}{2} \int S_{\left(s_{k}, t\right]} S^{2}(s) d\left\langle M^{c}\right\rangle_{s}\right\} \\
\cdot \prod_{s_{k}<s \leq t}\left\{1+A(s) \Delta b(s)+S(s) \Delta M_{s}\right\},
\end{gathered}
$$

where $b^{c}(t)$ is the continuous path of the function $b(t), s_{k} \leq t<s_{k+1}, k \geq 0$.

Let us notice that if

$$
A(t) \Delta b(t)+S(t) \Delta M_{t} \not \equiv-1
$$


on $\left(s_{k}, s_{k+1}\right)$, from the solution of (24) it follows

with some $T \in\left[s_{k}, \infty\right), k \geq 0$.

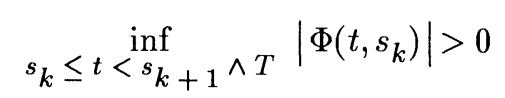

Let now define the function $\Phi(t), t \in \mathbb{R}_{+}$, where

$$
\Phi(t)=\Phi\left(t, s_{k}\right), s_{k} \leq t<s_{k+1}, k \geq 0 .
$$

It follows from(25) that the function $\Phi^{-1}(t), t \in \mathbb{R}_{+}$is correct defined and bounded on every finite interval $[0, T], T \in \mathbb{R}_{+}$. Consequently, for every $t \in \mathbb{R}_{+}$, it holds true that

$$
\int_{(0, t]} \Phi^{-2}(s) d\langle M\rangle_{s}<\infty .
$$

Theorem 3: The unique solution of the equation (22) is given by

$$
\begin{gathered}
X_{t}^{k}=\Phi\left(t, s_{k}\right)\left[X_{s_{k}}+\int_{\left(s_{k}, t\right]} \frac{\sigma(s)}{\Phi(s)} d M_{s}+\int_{\left(s_{k}, t\right]} \frac{a(s)}{\Phi(s)} d b(s)\right. \\
\left.-\int_{\left(s_{k}, t\right]} \frac{S(s) \sigma(s)}{\Phi(s)} d\left\langle M^{c}\right\rangle_{s}\right], s_{k} \leq t<s_{k+1}, k \geq 0 .
\end{gathered}
$$

Proof: Observe that (25) ensures that the process $X_{t}^{k}$ is well defined. We will show that the process $X_{t}^{k}$ from (27) is a solution of equation (22) over the interval $\left[s_{k}, s_{k+1}\right), k \geq 0$.

We apply Ito's rule to $(27)$ on the interval $\left(s_{k}, s_{k+1}\right)$ :

$$
\begin{gathered}
X_{t}^{k}=X_{s_{k}}+\int_{\left(s_{k}, t\right]} X_{s-} A(s) d b(s)+\int_{\left(s_{k}, t\right]} X_{s-} S(s) d M_{s} \\
+\int_{\left(s_{k}, t\right]} \frac{1}{1+A(s) \Delta b(s)+S(s) \Delta M_{s}}\left[\mathbf{I}_{\{\Delta b(s)=0\}}+\mathbf{I}_{\{\Delta b(s) \not \equiv\}]}\right. \\
\cdot\left[\sigma(s) d M_{s}+a(s) d b(s)-S(s) \sigma(s) \mathbf{I}_{\{\Delta b(s)=0\}} d\left\langle M^{c}\right\rangle_{s}\right]+\int_{\left(s_{k}, t\right]} S(s) \sigma(s) d\left\langle M^{c}\right\rangle_{s} \\
+\sum_{s_{k}<s \leq t}\left[1-\frac{1}{1+A(s) \Delta b(s)+S(s) \Delta M_{s}}\right]\left[\sigma(s) \Delta M_{s}+a(s) \Delta b(s)\right] ; \\
X_{t}^{k}=X_{s_{k}}+\int_{\left(s_{k}, t\right]} X_{s-} A(s) d b(s)+\int_{\left(s_{k}, t\right]} X_{s-} S(s) d M_{s} \\
+\int_{\left(s_{k}, t\right]} \sigma(s) d M_{s}+\int_{\left(s_{k}, t\right]} a_{a(s) d b(s) .}
\end{gathered}
$$

If $s_{k}<t<s_{k+1}$, then 


$$
X_{t}=X_{s_{k}}+\int_{\left(s_{k}, t\right]}\left[X_{s}-A(s)+a(s)\right] d b(s)+\int_{\left(s_{k}, t\right]}\left[X_{s}-S(s)+\sigma(s)\right] d M_{s} .
$$

The last representation and (23) lead to (22).

Remark 3: The coefficients $A(t), \sigma(t), S(t)$, and $a(t)$ of equation (22) are $f$-predictable functions. It will be convenient for applications to represent solution (27) in the form

$$
\begin{gathered}
X_{t}^{k}=\Phi\left(t, s_{k}\right)\left[X_{s_{k}}+\int_{\left(s_{k}, t\right]} \frac{\sigma(s)}{\Phi(s-)\left[1+A(s) \Delta b(s)+S(s) \Delta M_{s}\right]} d M_{s}\right. \\
+\int_{\left(s_{k}, t\right]} \frac{a(s)}{\Phi(s-)\left[1+A(s) \Delta b(s)+S(s) \Delta M_{s}\right]} d b(s) \\
\left.-\int_{\left(s_{k}, t\right]} \frac{S(s) \sigma(s)}{\Phi(s-)\left[1+A(s) \Delta b(s)+S(s) \Delta M_{s}\right]} d\left\langle M^{c}\right\rangle_{s}\right], \quad t \in\left[s_{k}, s_{k+1}\right), \quad k \geq 0 .
\end{gathered}
$$

\section{Acknowledgement}

The author would like to thank Professor Svetlozar Rachev from the UC Santa Barbara for his helpful and stimulating discussions and the referee for constructive comments.

\section{References}

[1] Aase, K.K., Contingent claim valuation when the security price is a combination of an Ito process and random point process, Stoch. Process. Appl. 28 (1988), 185-220.

[2] Cvitanić, J. and Karatzas, I., Hedging contingent claims with constrained portfolios, Ann. Appl. Probab. 3 (1993), 652-681.

[3] Doleans-Dade, C., Quelques applications de la formule de chagement de variables pour semimartingales, Z.W. 16 (1970), 181-194.

[4] Doleans-Dade, C., On the existence and unicity of solutions of stochastic integral equations, Z.W. 34 (1976), 93-101.

[5] Elliott, R.J., Stochastic Calculus and Applications, Springer-Verlag, Berlin 1982.

[6] Hadžiev, D., On the structure of Gaussian martingales, Serdica 4 (1978), 224-231 (in Russian).

[7] Karatzas, I. and Shreve, S.E., Brownian Motion and Stochastic Calculus, Springer-Verlag, New York 1987.

[8] Liptser, R.S. and Shiryaev, A.N., Martingale Theory, Nauka, Moscow 1986 (in Russian).

[9] Picqué, M. and Pontier, M., Optimal portfolio for a small investor in a market model with discontinuous prices, Appl. Math. Optim. 22 (1990), 287-310. 


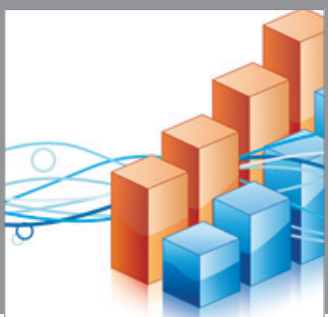

Advances in

Operations Research

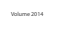

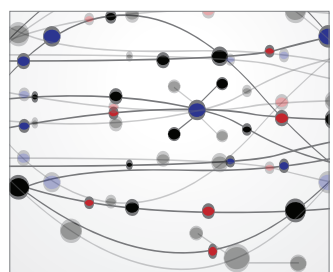

\section{The Scientific} World Journal
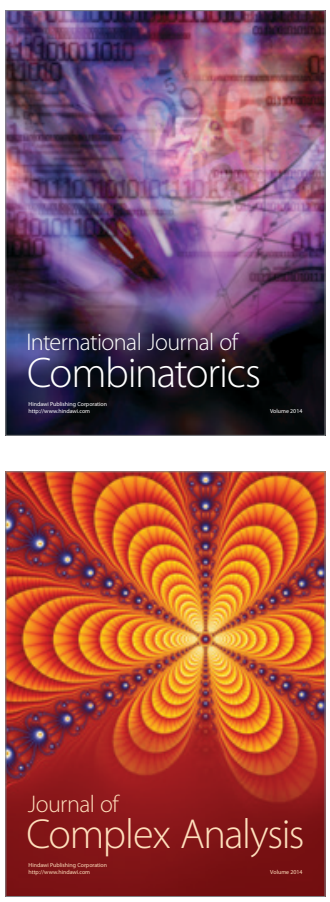

International Journal of

Mathematics and

Mathematical

Sciences
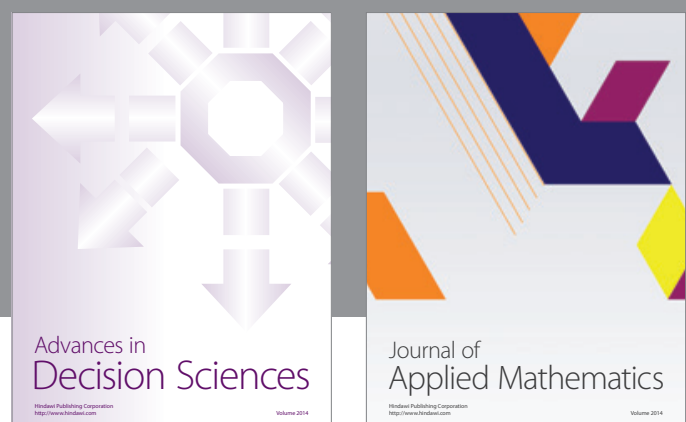

Journal of

Applied Mathematics
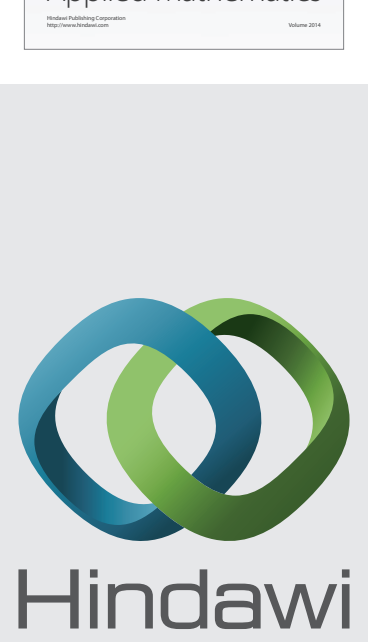

Submit your manuscripts at http://www.hindawi.com
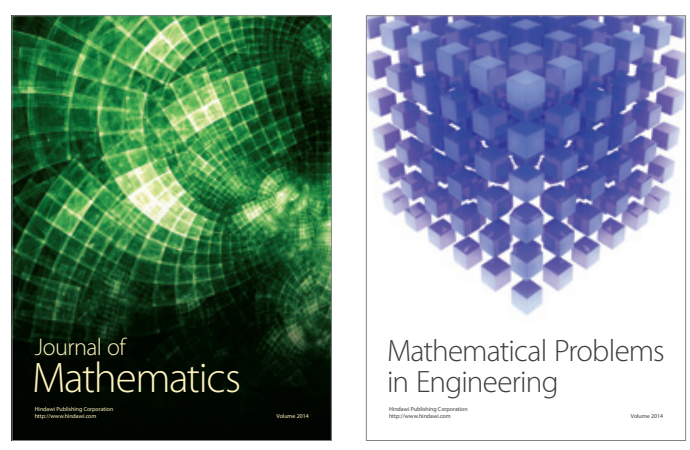

Mathematical Problems in Engineering
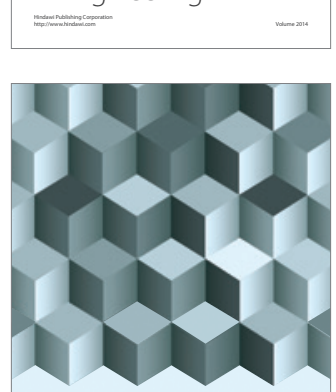

Journal of

Function Spaces
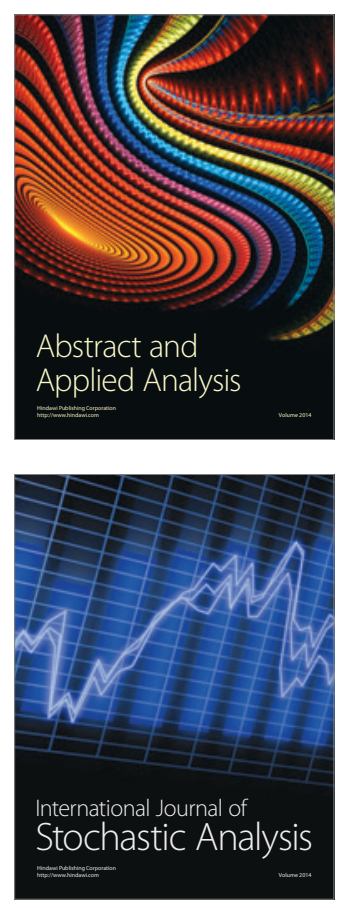

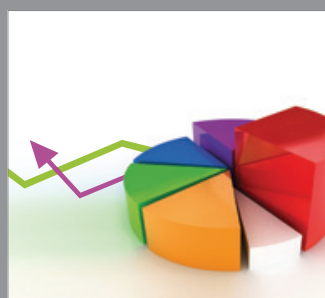

ournal of

Probability and Statistics

Promensencen
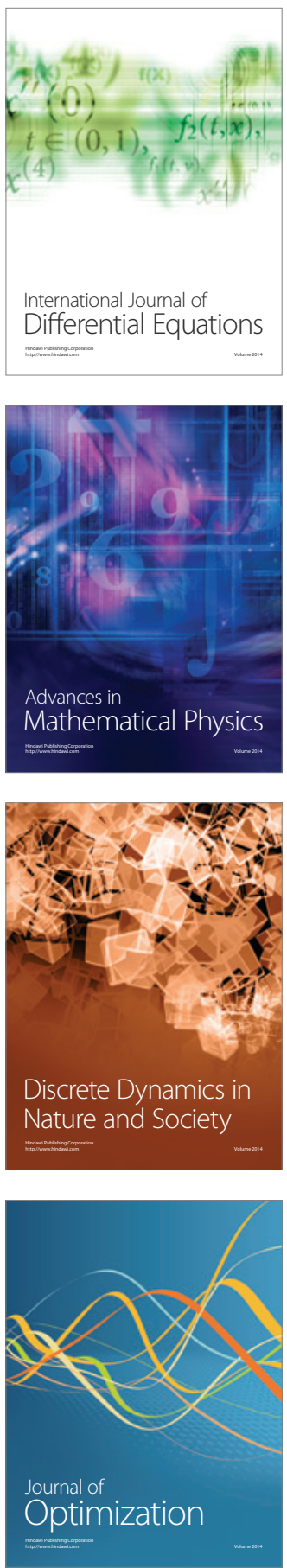